\title{
Clusters and Fluctuations at Mean-Field Critical Points and Spinodals
}

\author{
W. Klein,${ }^{\dagger}$ Harvey Gould,${ }^{ \pm}$J. Tobochnik,${ }^{\star}$ F. J. Alexander,${ }^{\S}$ M. Anghel,${ }^{\dagger}$ and Gregory Johnson ${ }^{ \pm}$ \\ ${ }^{\dagger}$ Department of Physics and Center for Computational Science, \\ Boston University, Boston, MA 02215 \\ ${ }^{ \pm}$Department of Physics, Clark University, Worcester, MA 01610 \\ ${ }^{\star}$ Department of Physics, Kalamazoo College, Kalamazoo, MI 49006 \\ ${ }^{\S}$ CIC-3, Los Alamos National Laboratory, Los Alamos, NM 87545
}

\begin{abstract}
We show that the structure of the fluctuations close to spinodals and mean-field critical points is qualitatively different than the structure close to non-mean-field critical points. This difference has important implications for many areas including the formation of glasses in supercooled liquids. In particular, the divergence of the measured static structure function in near-mean-field systems close to the glass transition is suppressed relative to the mean-field prediction in systems for which a spatial symmetry is broken.
\end{abstract}

The structure of the fluctuations near critical points and spinodals and their relation to the behavior of quantities observed in experiments and simulations is important for understanding the properties of many materials. For critical phenomena in non-mean-field Ising systems this relation was found by mapping the thermal critical point onto a percolation transition [1] 2]. In these systems the properties of the clusters at the percolation threshold are identical to those of the fluctuations at the thermal critical point. In particular, the mean cluster diameter is the correlation length, the density of the spanning cluster scales as the order parameter, and the mean number of sites in the spanning cluster is the susceptibility [2, 3].

For mean-field Ising models there is a line of spinodal critical points as well as the usual critical point. These mean-field thermal singularities also can be mapped onto percolation transitions [ 4 , but the relation between percolation clusters and critical fluctuations is qualitatively different. This difference has important consequences for supercooled mean-field liquids, which also exhibit spinodals [5], and for "near-mean-field" systems, which are characterized by long, but finite range interactions and which exhibit many of the characteristics of mean-field systems including pseudospinodals. Many dense systems with short-range interactions exhibit near-mean-field behavior under certain circumstances [4, 6, 7, 9].

In the following we give scaling arguments that relate the structure of clusters at mean-field critical points and spinodals to measurements of thermal quantities such as the static structure function $S(k)$ and discuss the implications of these results for understanding mean-field and near-mean-field supercooled liquids. One motivation for this analysis is that the behavior of a supercooled twocomponent, two-dimensional $(d=2)$ Lennard-Jones system has been interpreted as due to the influence of a pseudospinodal (relative to the stable solid) [7,8]. A consequence of this pseudospinodal is that the first peak of $S(k)$ is predicted to exhibit a power-law divergence, a prediction consistent with simulations in $d=2$ []]. However, experiments [10] and simulations [11] have failed to find similar behavior for $S(k)$ in $d=3$. This failure is puzzling because $d=3$ Lennard-Jones systems should be more mean-field-like than $d=2$ systems. This work addresses this apparent contradiction.

We first review how the cluster structure relates to thermal critical phenomena in non-mean-field systems. In mapping the percolation transition onto the thermal critical point, the quantity that is isomorphic to the singular part of the free energy is the mean number of clusters in a correlation region of volume $\xi^{d}$, where $\xi$ is the correlation length [2]. The singular part of the free energy scales as $\xi^{d} \epsilon^{2-\alpha}=\epsilon^{2-\alpha-d \nu}$, where $\epsilon=\left(T-T_{c}\right) / T_{c}$, and $\alpha$ and $\nu$ are the specific heat and correlation length exponents respectively [12]. Because hyperscaling is valid, we have $d \nu=2-\alpha$, and hence the mean number of clusters in a volume $\xi^{d}$ is order unity [2].

To obtain the scaling of the isothermal compressibility or susceptibility $\chi_{T}$, we use the relation of $\chi_{T}$ to the spatial integral of $\Gamma(r)$, the order parameter correlation function [13]. We take $\Gamma(r)$ to be the square of the order parameter over a volume $\xi^{d}$ and zero outside, and obtain $\chi_{T} \sim \epsilon^{2 \beta} \xi^{d}=\epsilon^{2-\alpha-d \nu-\gamma}$ using $\alpha+2 \beta+\gamma=2$ [12. Hyperscaling gives $2-\alpha-d \nu=0$ and hence $\chi_{T} \sim \epsilon^{-\gamma}$ as expected. This simple argument is well known, but fails in mean-field systems where hyperscaling is not valid.

In contrast to systems that obey hyperscaling, we need to distinguish between critical phenomena fluctuations and clusters in the mean-field limit. To discuss the latter we consider a weak long-range (Kac) interaction 14, 15 of the form $\gamma^{d} u(\gamma r)$, where $u(\gamma r)$ is integrable, a function only of the distance between spins, and ferromagnetic. If the system size is first taken to infinity and then the range of interaction $R=\gamma^{-1} \rightarrow \infty$, we obtain the Curie-Weiss description of the thermodynamics and the OrnsteinZernicke form for $\Gamma(r)$ 14,15]. The exponents for the mean-field critical point are $\nu=1 / 2, \alpha=0, \beta=1 / 2$ and $\gamma=1$ [12]. The singular part of the free energy at the mean-field critical point scales as

$$
\Delta f \sim \xi^{d} \epsilon^{2-\alpha}=R^{d} \epsilon^{2-d / 2} .
$$


The quantity $R^{d}$ appears because all lengths are in units of the interaction range.

From the percolation mapping [2, 何, Eq. (11) implies that the mean number of clusters in a volume $\xi^{d}$ is $R^{d} \epsilon^{2-d / 2}$. We can estimate the magnitude of this number from the Ginsburg criterion [16], which states that a system is well approximated by mean-field theory if $\chi_{T} / \phi^{2} \xi^{d}<<1$, where $\phi \sim \epsilon^{\beta}$ is the order parameter. If we use mean-field values for the critical exponents, we obtain the condition $R^{d} \epsilon^{2-d / 2}>>1$ for mean-field theory to be valid. This inequality, together with Eq. (1) and the percolation mapping, implies that the number of clusters in a volume $\xi^{d}$ is much greater than unity for mean-field systems. What does this result imply for the nature of clusters in mean-field systems?

In non-mean-field systems which have one cluster in a volume $\xi^{d}$, the free energy cost of this cluster is $\sim \xi^{d} \epsilon^{2-\alpha}$. As discussed above, this cost is order unity in non-meanfield systems. In contrast, because $\Delta f>>1$ in meanfield systems (see Eq. (1)), $\epsilon^{2-\alpha}$ is not the free energy density of one cluster in mean-field. Rather, there are $R^{d} \epsilon^{2-d / 2}$ clusters in a volume $\xi^{d}$, and each cluster has a free energy cost of order unity. This free energy implies that the probability of a cluster is order unity.

It also is clear that $\epsilon^{\beta}$ is not the density of one cluster, because if it were, the spin density in a volume of order $\xi^{d}$ would be $\approx R^{d} \epsilon^{2-d / 2} \epsilon^{\beta}$. For a fixed value of $\epsilon, R$ can be made arbitrarily large and hence this density can be arbitrarily large, an absurd result. This argument and the one for $\Delta f$ implies that $\epsilon^{\beta}$ is the density of all the spins in a volume $\xi^{d}$ regardless of the cluster to which they belong. It also implies that the density of spins in one cluster near the mean-field critical point [17] is

$$
\phi_{\mathrm{cl}} \sim \frac{\epsilon^{1 / 2}}{R^{d} \epsilon^{2-d / 2}} .
$$

This prediction has been verified numerically [18 19].

Are these clusters the critical phenomena fluctuations? To answer this question we calculate the density of meanfield fluctuations. The partition function for the Gaussian approximation of the $\phi^{4}$ model is given by 13

$$
Z=\int \delta \phi \exp \left\{-\beta \int d \vec{x} R^{d}\left[(\nabla \phi(\vec{x}))^{2}+\epsilon \phi^{2}(\vec{x})\right]\right\},
$$

where $\beta=1 / k_{B} T$ and $k_{B}$ is Boltzmann's constant. Because we are interested only in the scaling properties of the fluctuations, we take the order parameter $\phi(\vec{x})$ to be a constant $\phi$ over a volume $\xi^{d}$ and zero outside, and obtain $Z \sim \int \delta \phi e^{-\beta R^{d} \epsilon^{1-d / 2} \phi^{2}}$. If we integrate $\phi$ until the argument of the exponent becomes order unity, we find that the density of the critical phenomena fluctuations scales as

$$
\phi \sim \frac{\epsilon^{1 / 2}}{\left(R^{d} \epsilon^{2-d / 2}\right)^{1 / 2}} .
$$

That is, the structures with the density in Eq. (14) have a free energy cost of one. As in the non-mean-field case, we expect these objects to be the critical fluctuations.

From Eqs. (2) and (4) we see that the critical phenomena fluctuations are not the clusters, but are considerably denser in the mean-field limit $R^{d} \epsilon^{2-d / 2}>>1$. The structure of the critical phenomena fluctuations in mean-field systems is that the "vacuum" is not featureless but contains a very large number $\left(R^{d} \epsilon^{2-d / 2}\right.$ in a volume $\left.\xi^{d}\right)$ of clusters. At the critical point (magnetic field $h=0$ in Ising models), the mean number of up and down clusters is equal giving a zero mean magnetization. Because these clusters are independent [1,2], the fluctuations in the number of clusters is order $\left(R^{d} \epsilon^{2-d / 2}\right)^{1 / 2}$. These fluctuations make up the zero magnetization background and are the critical phenomena fluctuations. If we use Eq. (4), $\chi_{T} \sim \phi^{2} \xi^{d} \sim \epsilon R^{d} \epsilon^{-d / 2} / R^{d} \epsilon^{2-d / 2}=\epsilon^{-1}$ as expected.

These arguments can be extended to spinodals in mean-field Ising models. If the spinodal is approached by varying $h$ and keeping $T$ fixed, the density of the clusters is $\Delta h^{1 / 2} / R^{d} \Delta h^{3 / 2-d / 4}$ with $\Delta h=h_{s}-h$ [20]. The numerator represents the magnetic field scaling of the order parameter. The denominator is very large in mean-field and is the Ginsburg criterion for spinodals [21]. As at the critical point, the mean number of up and down clusters is equal when the infinite cluster, which is related to the metastable magnetization, is subtracted. All scaling arguments for mean-field critical points apply at spinodals with appropriate changes in the values of the exponents $(\nu=1 / 4, \alpha=1 / 2, \beta=1 / 2$, and $\gamma=1 / 2$ [20]).

We can determine the decay time of the clusters and the critical phenomena fluctuations by constructing an action from the linearized Langevin equation describing the dynamics of an Ising model with long-range interactions [22]. If we assume a random Gaussian noise, the probability measure for the order parameter $\phi(\vec{x}, t)$ is 23]

$$
\begin{array}{r}
\exp \left\{-\beta \int d \vec{x} d t\left[\epsilon\left(\frac{\partial \tilde{\phi}(\vec{x}, t)}{\partial t}\right)^{2}+\left(M R^{d} \epsilon^{2-d / 2}\right)^{2}\right.\right. \\
\left.\left.\left\{-\nabla^{2} \tilde{\phi}(\vec{x}, t)+\epsilon \tilde{\phi}(\vec{x}, t)\right\}^{2}+H(\bar{\psi}, \psi)\right]\right\},
\end{array}
$$

where $M$ is a (constant) mobility, $\tilde{\phi}=\epsilon^{1 / 2} \phi$, and $H(\bar{\psi}, \psi)=\bar{\psi}(\vec{x}, t)\left[\frac{\partial}{\partial t}+M R^{d} \epsilon^{2-d / 2}\left(-\nabla^{2}+\epsilon\right\}\right) \psi(\vec{x}, t)$. The variables $\bar{\psi}(\vec{x}, t)$ and $\psi(\vec{x}, t)$ obey a Grassmann algebra [22,23] and can be used to convert the average over the Gaussian noise to an average over the order parameter [22]. In the mean-field limit, the Langevin equation describing the dynamics is linear, and the Grassmann fields and the order parameter are independent [23]. Hence, the Grassmann fields are irrelevant to a calculation of averages of functions of $\phi$. Using the action in Eq. (5), we can average a function of $\phi$ by functionally integrating $\phi$ up to its value at the critical point where we expect that the argument of the exponential in Eq. (5) is order one. Because all of the terms in the action in 
Eq. (5) are real and positive, each term in the exponential must be order one, which implies that $\epsilon \xi^{d} \tilde{\phi}^{2} / t \sim 1$. Hence, the time scale that an object lives depends on $\tilde{\phi}$.

For critical fluctuations, $\tilde{\phi} \sim\left(R^{d} \epsilon^{2-d / 2}\right)^{-1 / 2}$, which leads to $\tau \sim \epsilon^{-1}$, the scaling for the decorrelation time in a mean-field system with a non-conserved order parameter. For clusters, $\tilde{\phi}_{\mathrm{cl}} \sim\left(R^{d} \epsilon^{2-d / 2}\right)^{-1}$, which leads to $\tau_{\mathrm{cl}} \sim \epsilon^{-1} / R^{d} \epsilon^{2-d / 2}$. We stress that these arguments are valid only in the mean-field limit and are a good approximation in the near-mean-field case.

These considerations imply that the clusters have a lifetime that is considerably shorter than the lifetime of the critical phenomena fluctuations. In the mean-field limit, $R^{d} \epsilon^{2-d / 2} \rightarrow \infty$, the lifetime of the clusters is zero.

We now apply these ideas to a mean-field model of a supercooled fluid. We consider the step potential $u(\gamma r)=$ 0 for $\gamma r>1$ and $u(\gamma r)=1$ for $\gamma r \leq 1$ [24]. For $\gamma \rightarrow 0$, $S(k)=1 /[1+\rho \beta u(k)]$ in the fluid phase [5], where $\rho$ is the density and $u(k)$ is the Fourier transform of $\gamma^{d} u(x)$ with $x$ and $k^{-1}$ scaled by $\gamma$. This mean-field form of $S(k)$ indicates that the system is unstable for $\rho \beta$ such that $1+\rho \beta_{s} u\left(k_{0}\right)=0$, where $u\left(k_{0}\right)<0$ is the minimum of $u(k)$. As $T \rightarrow T_{s}=\left(k_{B} \beta_{s}\right)^{-1}, S\left(k_{0}\right) \sim \epsilon^{-1}$ [0], where $\epsilon=\left|T-T_{s}\right| / T_{s}$. The divergence of $S\left(k_{0}\right)$ is unchanged if a short-ranged reference potential is added.

The scaling laws for the spinodal can be rewritten with $\epsilon$ as the scaling variable [25] with $\nu=1 / 2,2-\alpha=3$, $\beta=1$, and $\gamma=1$ rather than the values quoted previously for the $\Delta h$ scaling field [26]. The number of clusters in a volume $\xi^{d}$ scales as $R^{d} \epsilon^{3-d / 2}$, the density of clusters as $\epsilon / R^{d} \epsilon^{3-d / 2}$, and the density of critical phenomena fluctuations as $\epsilon /\left(R^{d} \epsilon^{3-d / 2}\right)^{1 / 2}$. The Ginsburg criterion is $\Lambda=R^{d} \epsilon^{3-d / 2}>>1$, and the time scale for the critical fluctuations and the clusters is $\epsilon^{-1}$ and $\epsilon^{-1} / R^{d} \epsilon^{3-d / 2}$, respectively.

The primary difference between the Ising and fluid spinodals is that $S(k)$ diverges at $k_{0} \neq 0$ for the fluid [5]. The Fourier transform of $S(k)$ yields a correlation function $\Gamma(r)$ that scales as $r^{-1} e^{-r / \xi} \sin k_{0} r$ in $d=3$, implying that the critical fluctuations near the spinodal have a characteristic length $k_{0}^{-1}<<\xi$ on which there is a periodic spatial variation. Since the critical fluctuations are an incoherent superposition of $\Lambda$ overlapping clusters, the spatial symmetry breaking reflected in $\Gamma(r)$ and $S(k)$ also occurs for the clusters. We can show from an analysis of the Langevin equation that the clusters have a triangular structure in $d=2$ and a bcc or layered triangular structure in $d=3$ [25]. (Nucleation near the spinodal is a coalescence of clusters [21] and the nucleation droplets have the symmetries discussed above [27,9].)

To understand the consequences of our interpretation of mean-field and near-mean-field fluctuations, we obtain the $T$-dependence of $S\left(k_{0}\right)$ for a fluid using scaling arguments similar to the ones used above. The structure function is related to an integral over the particles in a cluster times the probability that two particles belong to the same cluster within a critical fluctuation. The integral involves a phase factor $\sum_{j} \exp \left[i \vec{k}_{0, j} \cdot \vec{r}_{j}\right]$, where $\left|\vec{k}_{0, j}\right|=k_{0}$ and the index $j$ labels the basis reciprocal lattice directions for the indicated symmetry. This phase factor is multiplied by the probability that a site belongs to a critical phenomena fluctuation, $\epsilon /\left(R^{d} \epsilon^{3-d / 2}\right)^{1 / 2}$, times the probability that the second particle belongs to the same cluster, $\epsilon /\left(R^{d} \epsilon^{3-d / 2}\right)$, times the number of clusters, $\left(R^{d} \epsilon^{3-d / 2}\right)^{1 / 2}$. In addition, we average over the angles corresponding to the random orientations of the clusters. These considerations yield the scaling form:

$$
\begin{aligned}
S\left(k_{0}\right) & \sim \int d \vec{k}_{0}^{\prime} d \vec{r} \frac{\epsilon^{2}}{R^{d} \epsilon^{3-d / 2}} e^{i \vec{k}_{0} \cdot \vec{r}} \\
& \sim \frac{\epsilon^{2}}{R^{d} \epsilon^{3-d / 2}} \xi \sim R^{1-d} \epsilon^{-(3-d) / 2} .
\end{aligned}
$$

The spatial integral is over a region the size of $\xi^{d}$ and $d \vec{k}_{0}^{\prime}$ denotes an integral over angles.

Eq. (6) predicts that $S\left(k_{0}\right) \sim \epsilon^{-\tilde{\gamma}}$ with $\tilde{\gamma}=1$ in $d=1$, $\tilde{\gamma}=1 / 2$ in $d=2$, and $\tilde{\gamma}=0$ in $d \geq 3$ in contrast to $\gamma=1$ for all $d$ as predicted by mean-field theory [5]. We stress that the weakening of the divergence of $S\left(k_{0}\right)$ in $d=2$ and its supression for $d>2$ is a consequence of the quenched periodic structure of the clusters. If this structure is modified by finite size effects or defects, then the suppression might not be as strong.

The difference between the two calculations for the scaling behavior of $S\left(k_{0}\right)$ is the limiting procedure. If the limit $\Lambda \rightarrow \infty$ ia taken before the calculation of $S\left(k_{0}\right)$, the lifetime of the clusters is zero in comparison to a measurement time. In this limit the clusters appear rotationally symmetric and a calculation similar to Eq. (6) would yield $S\left(k_{0}\right) \sim \epsilon^{-1}$ for all $d$, the same result as in Ref. [5]. However, if we assume $\Lambda$ to be arbitrarily large but finite, the lifetime of the clusters is nonzero and the measurement time is smaller than the cluster lifetime. This assumption is consistent with the way measurements are done in experiments and simulations.

It is difficult to estimate critical exponents by fitting data directly to the suggested asymptotic form. However, because the spinodal is well defined only in the meanfield limit and simulations can be done only for finite $R$, we must estimate $\tilde{\gamma}$ by approaching the pseudospinodal. But we cannot approach it too closely because we will reach the Becker-Döring limit where the system nucleates very quickly. Because the Metropolis algorithm becomes very inefficient in near-mean-field because almost all single particle moves are accepted, we included a small hard core of diameter $\sigma$ to decrease the acceptance probability.

Our Monte Carlo results (see Fig. 1) in $d=1$ were fit to $A T^{\tilde{\gamma}} \epsilon^{-\tilde{\gamma}}$ with $\tilde{\gamma}=1.0 \pm 0.05$ and $T_{s} \approx 0.83$. The $T^{\tilde{\gamma}}$ term accounts in part for corrections to scaling which typically depend on $d$. The errors were estimated from different possible fits. The $d=2$ results were fit to $A \epsilon^{-\tilde{\gamma}}$ with $\tilde{\gamma}=0.4 \pm 0.05$ and $T_{s} \approx 0.81$. The quality of the 


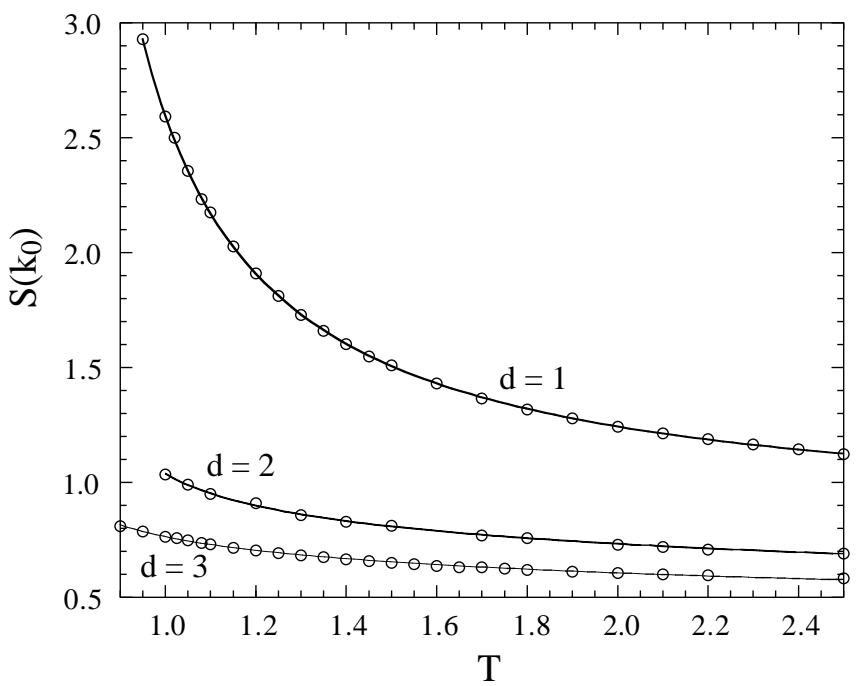

FIG. 1. The $T$-dependence of $S\left(k_{0}\right)$ in $d=1,2$, and 3 for the step potential discussed in the text. The $d=1$ results are for $N=10^{4}$ particles, interaction range $R=48$, hard core diameter $\sigma=0.05$, and density $\rho=1.95$. The fit shown for $S\left(k_{0}\right)$ is to $A T^{\tilde{\gamma}} \epsilon^{-\tilde{\gamma}}$ with $\tilde{\gamma}=1.02$ and $T_{s}=0.82$. For comparison, $T_{s}=0.85$ in the $R \rightarrow \infty$ limit. The $d=2$ results are for $N=10^{3}, R=6, \sigma=0.15$, and $\rho=1.95$. The fit for $S\left(k_{0}\right)$ is $A \epsilon^{-\tilde{\gamma}}$ with $\tilde{\gamma}=0.44$ and $T_{s}=0.81$. For comparison, $T_{s}=0.80$ for $R \rightarrow \infty$. The number of Monte Carlo steps per particle (MCS) for each value of $T$ is $10^{5}$ in $d=1$ and 2 . The $d=3$ results are for $N=10^{3}, R=3, \sigma=0.45$, and $\rho=1.95$ and fit equally well to $A \epsilon^{-\tilde{\gamma}}$ with $\tilde{\gamma}=0.16$ and $T_{s}=0.71$ and to $A \log \epsilon+B$ with $T_{s}=0.86 ; T_{s}=0.70$ for $R \rightarrow \infty$. The two fits are indistinguishable in the figure. The number of MCS for each data point is between $2 \times 10^{5}$ and $4 \times 10^{5}$.

fits is not as good as in $d=1$, but the estimate for $\tilde{\gamma}$ is consistent with our prediction of $\tilde{\gamma}=0.5$. The $d=3$ results are consistent with $A \epsilon^{-\tilde{\gamma}}$ with $\tilde{\gamma}=0.16 \pm 0.02$ and $T_{s} \approx 0.71$ and with $A \log \epsilon+B$ with $T_{s} \approx 0.86$. Details of the simulations will be discussed in a longer paper [25].

In summary, our theoretical and simulation results imply that the structure of the fluctuations in mean-field and near-mean-field systems differs qualitatively from that of non-mean-field systems. This structure leads to a suppression of the divergence of the measured static structure function near a pseudospinodal relative to the mean-field prediction in systems for which a spatial symmetry is broken. The dependence on $d$ of this suppression is such that there is no apparent divergence for $d \geq 3$ subject to logarthmic corrections. In these systems there is a growing correlation length as the pseudospinodal is approached that cannot be obtained from a measurement of $S(k)$. We note that a divergent dynamical length has been found above the glass transition in the spherical $p$ spin model 29]. This work, coupled with our results, raises the question of how the spinodal influences the dynamical length. Our predictions have important implications for the understanding of processes such as nucleation and glass formation in supercooled fluids. In par- ticular, we expect that the existence of clusters should help us understand the universal scaling behavior found for the dielectric response of organic glass formers [30].

We thank R. C. Brower, Bulbul Chakraborty, and John Rundle for useful discussions. This work was supported by NSF grant DMR-9633385 (WK and HG) and DOE DE-FG02-95ER 14498 (WK and MA). Acknowledgment is made to the donors of the Petroleum Research Foundation, administrated by the American Chemical Society, for partial support of the research at Kalamazoo College. Work on LA-UR 00-117 at Los Alamos National Laboratory was supported by the U.S. DOE LDRD-DR 98605.

[1] P. W. Kastaleyn and C. M. Fortuin, J. Phys. Soc. Jpn. (Suppl) 26, 11 (1969).

[2] A. Coniglio and W. Klein, J. Phys. A 13, 2775 (1980).

[3] D. Stauffer and A. Aharony, Introduction to Percolation Theory, Taylor \& Francis, London, 1992.

[4] W. Klein, Phys. Rev. Lett. 65, 1462 (1990).

[5] N. Grewe and W. Klein, J. Math. Phys. 18, 1735 (1977).

[6] D. W. Heermann, W. Klein and D. Stauffer, Phys. Rev. Lett. 49, 1262 (1982).

[7] G. Johnson et al., Phys. Rev. E 57, 5707 (1998).

[8] A spinodal-like limit to stability of supercooled fluids was first proposed by J. G. Kirkwood. See Phase Transformations in Solids, R. Smoluchowski, J. E. Mayer, and W. A. Wey, eds. (Wiley, New York, 1951).

[9] J. Yang et al., J. Chem. Phys. 93, 711 (1990).

[10] R. L. Leheny et al., J. Chem. Phys. 105, 7783 (1996).

[11] G. Johnson et al., in preparation.

[12] H. E. Stanley, Introduction to Phase Transitions and Critical Phenomena, Oxford, New York (1971).

[13] S.-k. Ma, Modern Theory of Critical Phenomena, Benjamin, Reading MA (1976).

[14] M. Kac, G. E. Uhlenbeck and P. Hemmer, J. Math. Phys. 4, 216 (1963).

[15] J. L. Lebowitz and O. Penrose, J. Math. Phys. 7, 98 (1965).

[16] L. D. Landau and E. M. Lifshitz, Statistical Physics, Vol. 1, third edition, translated by J. B. Sykes and M. J. Kearsley (Pergamon, NY 1980).

[17] Clusters with the same density have been found near spinodals of models of earthquake faults with long-range stress transfer. See M. Anghel et al., cond-mat/0002459.

[18] T. Ray and W. Klein, J. Stat. Phys. 53, 773 (1988).

[19] F. J. Alexander and W. Klein, in preparation.

[20] W. Klein and C. Unger, Phys. Rev. B 28, 445 (1983).

[21] L. Monette and W. Klein, Phys. Rev. Lett. 68, 2336 (1992).

[22] G. Parisi and N. Sourlas, Phys. Rev, Lett. 43, 744 (1979).

[23] W. Klein and G. G. Batrouni, Phys. Rev. Lett. 67, 1278 (1991).

[24] W. Klein et al., Physica A 205, 738 (1994).

[25] W. Klein et al., in preparation. 
[26] The proper scaling field for the spinodal is $a \Delta h+b \epsilon$, where $a$ and $b$ are constants.

[27] W. Klein and F. Leyvraz, Phys. Rev. Lett. 57, 2485 (1986).

[28] D. W. Heermann, W. Klein, and D. Stauffer, Phys. Rev. Lett. 49, 1262 (1982).

[29] C. Donati et al., cond-mat/9905433.

[30] P. K. Dixon et al., Phys. Rev. Lett. 65, 1108 (1990). 